\title{
Effects of different serum conditions on osteogenic differentiation of human adipose stem cells in vitro
}

Laura Kyllönen ${ }^{1,2,3+}$, Suvi Haimi ${ }^{1,2,3,4^{*}+}$, Bettina Mannerström ${ }^{1,2,3}$, Heini Huhtala ${ }^{5}$, Kristiina M Rajala²,3,6, Heli Skottman ${ }^{2,3,7}$, George K Sándor ${ }^{1,2,3,8}$ and Susanna Miettinen ${ }^{1,2,3}$

\begin{abstract}
Introduction: Currently, human adipose stem cells (hASCs) are differentiated towards osteogenic lineages using culture medium supplemented with L-ascorbic acid 2-phosphate (AsA2-P), dexamethasone (Dex) and betaglycerophosphate ( $\beta-G P)$. Because this osteogenic medium (OM1) was initially generated for the differentiation of bone marrow-derived mesenchymal stem cells, the component concentrations may not be optimal for the differentiation of hASCs. After preliminary screening, two efficient osteogenic media (OM2 and OM3) were chosen to be compared with the commonly used osteogenic medium (OM1). To further develop the culture conditions towards clinical usage, the osteo-inductive efficiencies of $\mathrm{OM} 1, \mathrm{OM} 2$ and $\mathrm{OM} 3$ were compared using human serum (HS)-based medium and a defined, xeno-free medium (RegES), with fetal bovine serum (FBS)-based medium serving as a control.

Methods: To compare the osteo-inductive efficiency of OM1, OM2 and OM3 in FBS-, HS- and RegES-based medium, the osteogenic differentiation was assessed by alkaline phosphatase (ALP) activity, mineralization, and expression of osteogenic marker genes (runx2A, DLX5, collagen type I, osteocalcin, and ALP).

Results: In HS-based medium, the ALP activity increased significantly by OM3, and mineralization was enhanced by both $\mathrm{OM} 2$ and $\mathrm{OM} 3$, which have high AsA2-P and low Dex concentrations. ALP activity and mineralization of hASCs was the weakest in FBS-based medium, with no significant differences between the OM compositions due to donor variation. However, the qRT-PCR data demonstrated significant upregulation of runX2A mRNA under osteogenic differentiation in FBS- and HS-based medium, particularly by OM3 under FBS conditions. Further, the expression of DLX5 was greatly stimulated by $\mathrm{OM} 1$ to 3 on day 7 when compared to control. The regulation of collagen type I, ALP, and osteocalcin mRNA was modest under induction by OM1 to 3. The RegES medium was found to support the proliferation and osteogenic differentiation of hASCs, but the composition of the RegES medium hindered the comparison of OM1, OM2 and OM3.

Conclusions: Serum conditions affect hASC proliferation and differentiation significantly. The ALP activity and mineralization was the weakest in FBS-based medium, although osteogenic markers were upregulated on mRNA level. When comparing the OM composition, the commonly used OM1 was least effective. Accordingly, higher concentration of AsA2-P and lower concentration of Dex, as in $\mathrm{OM} 2$ and $\mathrm{OM} 3$, should be used for the osteogenic differentiation of hASCs in vitro.
\end{abstract}

\footnotetext{
* Correspondence: suvi.haimi@uta.fi

+ Contributed equally

'Adult Stem Cells Group, Institute of Biomedical Technology, University of

Tampere, Biokatu 8, Tampere Fl-33014, Finland

Full list of author information is available at the end of the article
} 


\section{Introduction}

The osteogenic potential of human adipose stem cells (hASCs) has recently stimulated interest in clinical bone tissue engineering [1,2]. This multipotent population of cells, isolated from the stromal vascular compartment of adipose tissue, was originally characterized by Zuk and co-workers [3]. It was soon discovered that hASCs are able to differentiate toward osteogenic, adipogenic, myogenic, and chondrogenic lineages in vitro, when treated with appropriate inducing factors [3]. Since their discovery, different approaches have been developed to enhance osteogenic capacity of hASCs. Much of the research has concentrated on the osteo-induction of hASCs via growth factors such as bone morphogenetic proteins (BMPs) [4-6]. Although considerable research has been devoted to BMPs, their cost-effectiveness and safety in clinical use have been under controversy [7-10]. Recent studies have also questioned whether hASCs are responsive to BMPs at all [11]. Therefore, efficient methods for osteo-induction of hASCs are still required.

Commonly, osteogenic differentiation of mesenchymal stem cells (MSCs) in in vitro culture has been managed by supplementing the growth medium with $50 \mu \mathrm{M} \mathrm{L}$ ascorbic acid 2-phosphate (AsA2-P), $100 \mathrm{nM}$ dexamethasone (Dex) and $10 \mathrm{mM}$ beta-glycerophosphate ( $\beta$ GP) [11-14]. Because this osteogenic medium (OM) was initially generated for the differentiation of bone marrow-derived mesenchymal stem cells (BMSCs) [15], the component concentrations may not be optimal for the differentiation of hASCs [16]. Although BMSCs and ASCs possess many similar characteristics, their response to inductive stimuli may not be identical $[12,17,18]$. Whereas the combined and separate effects of Dex and AsA2-P have been largely studied with BMSCs $[15,19-21]$, there are only limited studies with hASCs $[16,22]$. For example, de Girolamo and co-workers suggested that OM with lower Dex and higher AsA2-P concentration could be more effective with hASCs than the commonly used OM [16]. However, this study was conducted using two donor cell lines and more importantly only fetal bovine serum (FBS)-containing medium. Therefore, we conducted a preliminary screening using different osteogenic supplement concentrations in human serum (HS)-based medium. Based on these preliminary results, two efficient compositions (OM2 and OM3) were chosen for further comparison in different serum conditions with the commonly used $\mathrm{OM}$ (referred here as OM1).

So far, the effects of OM on hASC differentiation have been defined mostly using FBS-containing medium $[12,13,16]$. While FBS-based medium may be acceptable for the in vitro experiments, exposure to undefined animal-derived products poses a risk in clinical stem cell therapies [23,24]. Replacing animal-derived products such as FBS with human serum, human platelet lysate, platelet-rich plasma or defined xeno-free alternative, significantly enhances the safety and quality of stem cells for therapeutic approaches $[23,25]$. In addition to safety issues, serum conditions can have a significant effect on stem cell characteristics such as differentiation capacity and proliferation [26,27]. In order to define how serum conditions affect the osteogenic differentiation of hASCs, we tested HS-based medium and a defined, xeno-free medium (RegES) in comparison to FBS-based medium to evaluate the efficiency of three different OM compositions. The fully defined xeno-free medium formulation RegES was previously developed in our institute for stem cell culture [28].

In order to produce clinically relevant in vitro data the effects of three different OM compositions on hASCs were compared in the present study using FBS, HS and xeno-free RegES medium.

\section{Materials and methods}

\section{Ethics statement}

This study was conducted in accordance with the Ethics Committee of the Pirkanmaa Hospital District, Tampere, Finland (R03058), to obtain adipose tissue samples for research purposes. The hASCs were isolated from adipose tissue samples acquired from surgeries performed in the Department of Plastic Surgery, Tampere University Hospital. The subcutaneous adipose tissue samples harvested from either abdomen or breast were obtained with a written informed consent from six female donors (mean age $53 \pm 16$ ).

\section{Isolation and cell culture}

Human ASCs were isolated from adipose tissue samples by mechanical and enzymatic method as described previously $[26,29]$. Briefly, the adipose tissue samples were minced into smaller pieces and digested with collagenase type I $(1.5 \mathrm{mg} / \mathrm{ml}$; Invitrogen, Carlsbad, CA, USA), followed by centrifugation and filtering steps. The isolated hASCs were maintained and expanded in maintenance medium (MM) consisting of Dulbecco's modified Eagle's medium/Ham's Nutrient Mixture F-12 (DMEM/ F-12 1:1; Invitrogen) supplemented with 1\% L-alanyl-Lglutamine (GlutaMAX; Invitrogen), $1 \%$ antibiotics (100 $\mathrm{U} / \mathrm{ml}$ penicillin, $0.1 \mathrm{mg} / \mathrm{ml}$ streptomycin; Invitrogen), and either 10\% allogeneic HS (PAA Laboratories GmbH, Pasching, Austria) (referred as HS MM) or 10\% FBS Gold (PAA Laboratories) (referred as FBS MM). FBS Gold was used for the experiments, because it has been reported to eliminate the need for extensive and timeconsuming batch testing due to its constant quality. The hASCs from each donor were isolated into both HS and 
FBS maintenance medium. The hASCs isolated and expanded in HS medium were detached using TrypLE Select (Life Technologies, Carlsbad, CA, USA), whereas hASCs in FBS medium were detached using 1\% trypsin (Lonza Biowhittaker, Verviers, Belgium). For xeno-free conditions, hASCs were first isolated and expanded in HS MM, and xeno-free RegES medium was added after hASCs were seeded onto well plates. The composition of RegES maintenance medium (RegES MM) is shown in Additional file 1. A CELLstart (Invitrogen) pre-coating of polystyrene well plates was required for the cell attachment under RegES conditions. The experiments were carried out at passages three to four.

\section{Characterization of the cells}

In order to verify the mesenchymal origin, hASCs must meet several criteria defined by the International Society for Cellular Therapy [30]. The mesenchymal origin of hASCs used in this study was confirmed by their adherence to plastic, differentiation capacity to osteogenic, chondrogenic and adipogenic lineages in vitro, and by their surface marker expression.

\section{Flow cytometric surface marker expression analysis}

Flow cytometry was used to characterize the surface marker expression of hASCs cultured in FBS and HS MM. Flow cytometric characterization comparing the surface marker expression of hASCs cultured in HS medium and xeno-free RegES medium has been reported previously [28]. Briefly, hASCs cultured in FBS and HS MM were analyzed by a fluorescence-activated cell sorter (FACSAria; BD Biosciences, Erembodegem, Belgium) as described by Lindroos and co-workers [26]. Monoclonal antibodies against CD14-PE, CD19-PE, CD49d-PE, CD73-PE, CD90-APC, CD106-PE, (BD Biosciences); CD45-FITC (Miltenyi Biotech, Bergisch Gladbach, Germany); CD34-APC, HLA-ABC-PE, HLA-DRPE (Immunotools GmbH Friesoythe, Germany); and CD105-PE (R\&D Systems Inc, Minneapolis, MN, USA) were used. Analysis was performed on 10,000 cells per sample and unstained cell samples were used to compensate for the background autofluorescence levels.

\section{Analysis of multipotent differentiation capacity}

The multipotent differentiation of hASCs was conducted in RegES medium supplemented with corresponding adipogenic, osteogenic and chondrogenic components. The multipotent differentiation capacity of hASCs cultured in FBS and HS medium has been shown previously by Lindroos and co-workers [31].

For multipotency analysis, CELLstart pre-coating was used for well plates in all differentiation procedures except for chondrogenic differentiation. For osteogenic induction analysis, hASCs were seeded on 12-well plates
Table 1 The medium compositions used in the study.

\begin{tabular}{ll}
\hline Medium & Composition \\
\hline $\begin{array}{ll}\text { Maintenance } \\
\text { medium (MM) }\end{array}$ & 10\% FBS-, 10\% HS-, or RegES-medium \\
\hline $\mathrm{OM} 1$ & $100 \mathrm{nM}$ Dex, $50 \mu \mathrm{M}$ AsA2P, $10 \mathrm{mM} \beta$-GP in MM \\
\hline $\mathrm{OM} 2$ & $10 \mathrm{nM} \mathrm{Dex}, 150 \mu \mathrm{M}$ AsA2P, $10 \mathrm{mM} \beta$-GP in MM \\
\hline $\mathrm{OM} 3$ & $5 \mathrm{nM}$ Dex, $250 \mu \mathrm{M}$ AsA2P, $10 \mathrm{mM} \beta$-GP in MM \\
\hline
\end{tabular}

Maintenance medium (MM) was culture medium with $10 \%$ FBS or $10 \% \mathrm{HS}$, or defined, xeno-free RegES-medium (composition defined in Additional file 1). The osteogenic media (OM) were supplemented with dexamethasone (Dex), L-ascorbic acid 2-phosphate (AsA2-P), and beta-glycerophosphate ( $\beta$-GP). MM, maintenance medium; OM, osteogenic medium; FBS, fetal bovine serum; HS, human serum; Dex, dexamethasone; AsA2-P, L-ascorbic acid 2-phosphate; $\beta$ GP, beta-glycerophosphate.

at a density of $2.5 \times 10^{3}$ cells $/ \mathrm{cm}^{2}$ in HS MM. After 24 hours, the HS medium was replaced by OM3 in RegES medium (Table 1). The osteogenic differentiation was detected by alkaline phosphatase (ALP) staining at a 14day time point. Briefly, cell cultures were fixed with $4 \%$ paraformaldehyde (PFA) and stained with a leukocyte ALP kit according to Sigma procedure 86 (SigmaAldrich, St Louis, MO, USA).

For the adipogenic induction, hASCs were seeded on 12 -well plates at a density of $2 \times 10^{4}$ cells $/ \mathrm{cm}^{2}$ in HS MM. After 2 days, RegES medium with adipogenic supplements was added: $33 \mu \mathrm{M}$ biotin (Sigma-Aldrich), 1 $\mu \mathrm{M}$ Dex (Sigma-Aldrich), $100 \mathrm{nM}$ insulin (Life Technologies), and $17 \mu \mathrm{M}$ pantothenate (Fluka, Buchs, Switzerland). In addition to other adipogenic supplements, 250 $\mu \mathrm{M}$ isobutylmethylxanthine (IBMX; Sigma-Aldrich) was used for the first 24 hours of adipogenic induction. After 14 days of culture, the intracellular lipid accumulation was detected by Oil Red $\mathrm{O}$ staining. Cell cultures were fixed with $4 \%$ PFA, followed by treatment with $60 \%$ isopropanol, and stained with $0.5 \%$ Oil Red O solution (Sigma-Aldrich) in 60\% isopropanol.

A micromass culture technique was used for the chondrogenic differentiation, where $1 \times 10^{5}$ cells were seeded on 24-well plates in a $10 \mu \mathrm{l}$ volume, and allowed to adhere for 3 hours in $+37^{\circ} \mathrm{C}$ prior to the addition of RegES medium with chondrogenic supplements: $1 \%$ ITS +1 (Sigma-Aldrich), $50 \mu \mathrm{M}$ AsA2-P (Sigma-Aldrich), 55 $\mu \mathrm{M}$ sodium pyruvate (Life Technologies), $23 \mu \mathrm{M}$ L-proline (Sigma-Aldrich), $10 \mathrm{ng} / \mathrm{ml}$ transforming growth factor-beta (Sigma-Aldrich). The chondrogenic differentiation was confirmed by histological Alcian Blue (SigmaAldrich) staining after 14 days of culture. The micromass pellets were fixed in 4\% PFA, embedded in paraffin and sectioned at $5 \mu \mathrm{m}$ thickness. Alcian Blue ( $\mathrm{pH} 1.0)$ solution was used to detect sulphated glycosaminoglycans (GAGs) characteristic in cartilaginous matrices. Nuclear Fast Red (Biocare Medical, Concord, MA, USA) was used as a counterstain. 


\section{Osteogenic medium compositions}

For the comparison of the different OM compositions, hASCs were plated on 12-well plates at a density of $7 \times$ $10^{3}$ cells $/ \mathrm{cm}^{2}$. The cells were plated in either HS or FBS MM and allowed to attach for 24 hours before starting the osteogenic differentiation. For xeno-free conditions, the well plates were pre-coated with CELLstart and hASCs were plated in HS MM for 24 hours to facilitate attachment of the cells before medium was replaced by RegES MM or RegES with osteogenic supplements (Table 1). Three different osteogenic medium compositions, OM1, OM2 and OM3, were compared in FBS, HS and xeno-free conditions (Table 1). Based on the literature, OM with lower Dex and higher AsA2-P concentration than in the traditionally used OM (OM1) was suggested to be more optimal for osteo-induction of hASCs in FBS-based medium [16]. We conducted a preliminary screening to test different concentrations of AsA2-P and Dex in HS-based medium (Additional file 2). Based on these results, OM2 and OM3 with low Dex and high AsA2-P concentrations were chosen for further comparison with the traditionally used OM1. AsA2-P, Dex and $\beta$-GP used in the osteogenic media were all purchased from Sigma-Aldrich. The hASCs cultured in FBS MM were used as a reference in all the analyses. It must be noted that RegES MM contains a high basal level of AsA2-P $(50 \mu \mathrm{g} / \mathrm{ml}$ that corresponds to $170 \mu \mathrm{M})$. As a consequence, the additional AsA2-P in the osteogenic compositions raised the total concentrations of AsA2-P in RegES-based OM1, OM2 and OM3 to 220, 320 , and $420 \mu \mathrm{M}$, respectively.

\section{Morphology and cell number}

Cell number, based on the total amount of DNA per sample, was determined using a CyQUANT ${ }^{\mathrm{TM}}$ Cell Proliferation Assay Kit (CyQUANT; Molecular Probes, Invitrogen, Paisley, UK) as described previously [32]. Briefly, at 1-, 7- and 14-day time points the cells were lysed with $0.1 \%$ Triton-X 100 buffer (Sigma-Aldrich) and analyzed after a freeze-thaw cycle. Fluorescence was measured with a microplate reader (Victor 1420 Multilabel Counter; Wallac, Turku, Finland) at 480/520 $\mathrm{nm}$. Morphology of the cells was observed at 1-, 3-, 7- and 14-day time points using light microscopy.

\section{Alkaline phosphatase activity and mineralization}

The osteogenic differentiation capacity of hASCs was determined at 7 and 14 days by analyzing ALP activity and mineralization. ALP is a generally used marker for early osteogenic differentiation, whereas mineralization of the ECM is a characteristic of late osteogenic differentiation. The quantitative ALP analysis was performed on the same samples as the analysis of cell number, using the ALP Kit (Sigma-Aldrich) as reported previously [32]. A quantitative Alizarin Red S method was used at 7 and 14 days to detect mineralization as described previously [32]. Briefly, the ethanol fixed cells were stained with $2 \%$ Alizarin Red S solution (SigmaAldrich), and photographed after several steps of washing. Cetylpyridinium chloride (Sigma-Aldrich) was used to extract the dye, followed by absorbance measurement at $540 \mathrm{~nm}$ with a microplate reader (Victor 1420).

\section{Quantitative real-time PCR}

Quantitative real-time reverse transcription polymerase chain reaction (qRT-PCR) analysis was used to compare the relative expression of osteogenic genes under different culturing conditions. For qRT-PCR analysis, hASCs were seeded on 6 -well plates at a density of $7 \times 10^{3}$ cells $/ \mathrm{cm}^{2}$. A CELLstart pre-coating of well plates was used in xeno-free conditions. Total RNA was isolated from the cells at 7-and 14-day time points with Nucleospin kit reagent (Macherey-Nagel GmbH \& Co. KG, Düren, Germany) according to manufacturer's instructions. First-strand cDNA was synthesized from total RNA using the High-Capacity cDNA Reverse Transcriptase Kit (Applied Biosystems, Foster City, CA, USA). The expression of osteogenic genes including runx2A, DLX5, collagen type I, osteocalcin, and ALP was analyzed. Isoform A of runx 2 was analyzed due to its specificity for osteogenic differentiation in comparison to isoform C $[33,34]$. Data was normalized to the expression of RPLP0 (human acidic ribosomal phosphoprotein $\mathrm{P} 0$ ), a housekeeping gene, which has shown to have stable expression under different experimental conditions in similar studies [35,36]. The primer sequences (Oligomer Oy, Helsinki, Finland) and accession numbers are presented in Table 2. The qRT-PCR mixture contained $50 \mathrm{ng}$ cDNA, $300 \mathrm{nM}$ forward and reverse primers, and SYBR Green PCR Master Mix (Applied Biosystems). The reactions were conducted with AbiPrism 7000 Sequence Detection System (Applied Biosystems) with initial enzyme activation at $95^{\circ} \mathrm{C}$ for 10 minutes, followed by 45 cycles of denaturation at $95^{\circ} \mathrm{C}$ for 15 seconds and anneal and extend at $60^{\circ} \mathrm{C}$ for 60 seconds. The expression levels of all differentiation cultures were compared to the expression level of FBS control cultures.

\section{Statistical analysis}

Statistical analyses were performed with SPSS version 19 (IBM, Armonk, NY, USA). The effects of different culture conditions on cell number, normalized ALP activity, mineralization and relative gene expression were compared with nonparametric statistics using KruskalWallis one-way analysis of variance by ranks, with Mann-Whitney post hoc test to analyze the specific sample pairs for significant differences. The significances 
Table 2 The primer sequences for qRT-PCR.

\begin{tabular}{|c|c|c|c|c|}
\hline Name & & $5^{\prime}$ - Sequence $-3^{\prime}$ & Product size (bp) & Accession number \\
\hline \multirow[t]{2}{*}{ RUNX2A } & Forward & CTTCATTCGCCTCACAAACAAC & 62 & NM_001024630.3 \\
\hline & Reverse & TCCTCCTGGAGAAAGTTTGCA & & \\
\hline \multirow[t]{2}{*}{ DLX5 } & Forward & ACCATCCGTCTCAGGAATCG & 75 & NM_005221.5 \\
\hline & Reverse & CCCCCGTAGGGCTGTAGTAGT & & \\
\hline \multirow[t]{2}{*}{ Collagen type I } & Forward & CCAGAAGAACTGGTACATCAGCAA & 94 & NM_00088 \\
\hline & Reverse & CGCCATACTCGAACTGGAATC & & \\
\hline \multirow[t]{2}{*}{ Osteocalcin } & Forward & AGCAAAGGTGCAGCCTTTGT & 63 & NM_000711 \\
\hline & Reverse & GCGCCTGGGTCTCTTCACT & & \\
\hline \multirow[t]{2}{*}{ Alkaline phosphatase } & Forward & CCCCCGTGGCAACTCTATCT & 73 & NM_000478.4 \\
\hline & Reverse & GATGGCAGTGAAGGGCTTCTT & & \\
\hline \multirow[t]{2}{*}{$\overline{R P L P O}$} & Forward & AATCTCCAGGGGCACCAT T & 70 & NM_001002 \\
\hline & Reverse & CGCTGGCTCCCACTITGT & & \\
\hline
\end{tabular}

obtained were corrected using Bonferroni adjustment in order to justify multiple comparisons. For example, the obtained $P$ value was multiplied by the comparisons made within the time point (MM vs. OM1/OM2/OM3, OM1 vs. OM2/OM3, and OM2 vs. OM3 equals six comparisons within one time point), and multiplied with the number of time points $(6 \times 3$ time points $=18$, or 6 $\times 2$ time points $=12$ ). When for example $P=0.002$ was obtained with Mann-Whitney, the $P$ value was multiplied with 12 or 18 , giving the final $P$ values 0.024 or 0.036 respectively, depending the number of time points. The results were considered significant when $P<0.05$. The effect of culture duration on cell number was analyzed similarly using Kruskal-Wallis, Mann-Whitney and Bonferroni adjustment as described. All the results were standardized to the control condition (FBS MM). All the experiments were repeated three times using different donor in each repeat $(n=3)$. Technical triplicates of each sample were used in all the assays.

\section{Results}

\section{Flow cytometric surface marker expression analysis}

The flow cytometric analysis demonstrated that hASCs cultured in FBS and HS MM express the surface markers CD73, CD90, CD105 (Table 3). The hASCs lacked the expression of the CD14, CD19, HLA-DR, the hematopoietic marker $\mathrm{CD} 45$, and the vascular cell adhesion molecule CD106. The expression of CD34, CD49d and HLA-ABC was moderate. The results verified the mesenchymal origin of the hASCs, and the lack of hematopoietic and angiogenic markers [26,37,38].

\section{Analysis of multipotent differentiation capacity}

The multipotent differentiation capacity of the hASCs cultured in RegES was examined by culturing the cells under osteogenic, adipogenic and chondrogenic conditions (Figure 1A-C). Differentiation was analyzed by histological stainings at a 14-day time point. Osteogenic differentiation was confirmed by a positive ALP staining at day 14 (Figure 1A). Adipogenic differentiation (Figure 1B) was verified by Oil Red O staining, showing the lipid droplets present in differentiated cells. Chondrogenic differentiation (Figure 1C) was confirmed by the formation of aggregates as well as Alcian Blue staining. The chondrogenically induced hASCs formed aggregates that were intensively stained by Alcian Blue,

Table 3 Characterization of hASCs.

\begin{tabular}{|c|c|c|c|}
\hline Antigen & Surface protein & FBS & HS \\
\hline$\overline{\mathrm{CD} 14}$ & $\begin{array}{l}\text { Serum lipopolysaccharide- } \\
\text { binding protein }\end{array}$ & $2.6 \pm 2.7$ & $1.1 \pm 1.0$ \\
\hline CD19 & $\begin{array}{l}\text { B lymphocyte-lineage } \\
\text { differentiation antigen }\end{array}$ & $1.1 \pm 0.9$ & $0.4 \pm 0.1$ \\
\hline CD34 & $\begin{array}{l}\text { Sialomucin-like adhesion } \\
\text { molecule }\end{array}$ & $19.6 \pm 12.2$ & $27.4 \pm 23.5$ \\
\hline CD45 & Leukocyte common antigen & $1.5 \pm 0.8$ & $1.8 \pm 1.6$ \\
\hline CD49d & Integrin a2, VLA-4 & $12.7 \pm 2.5$ & $35.4 \pm 10.1$ \\
\hline $\mathrm{CD} 73$ & Ecto-50-nucleotidase & $82.2 \pm 8.5$ & $85.9 \pm 7.3$ \\
\hline CD90 & $\begin{array}{l}\text { Thy-1 ( } T \text { cell surface } \\
\text { glycoprotein) }\end{array}$ & $97.3 \pm 2.5$ & $96.5 \pm 4.6$ \\
\hline$\overline{C D 105}$ & $\mathrm{SH}-2$, endoglin & $85.3 \pm 17.7$ & $79.2 \pm 21.1$ \\
\hline CD106 & $\begin{array}{l}\text { VCAM-1 (vascular cell } \\
\text { adhesion molecule) }\end{array}$ & $0.6 \pm 0.3$ & $1.3 \pm 1.7$ \\
\hline HLA-ABC & $\begin{array}{l}\text { Major histocompatibility class } \\
\text { I antigens }\end{array}$ & $16.9 \pm 8.1$ & $28.1 \pm 16.4$ \\
\hline HLA-DR & $\begin{array}{l}\text { Major histocompatibility class } \\
\text { II antigens }\end{array}$ & $1.9 \pm 1.2$ & $1.0 \pm 1.0$ \\
\hline
\end{tabular}

Surface marker expression of undifferentiated hASCs cultured either in FBS- or HS-medium as analyzed by flow cytometric analysis. Data is presented as mean \pm standard deviation of the percentage of surface marker expression ( $n$ =6). hASCs, human adipose stem cells; FBS, fetal bovine serum; HS, human serum; $C D$, cluster of differentiation. 


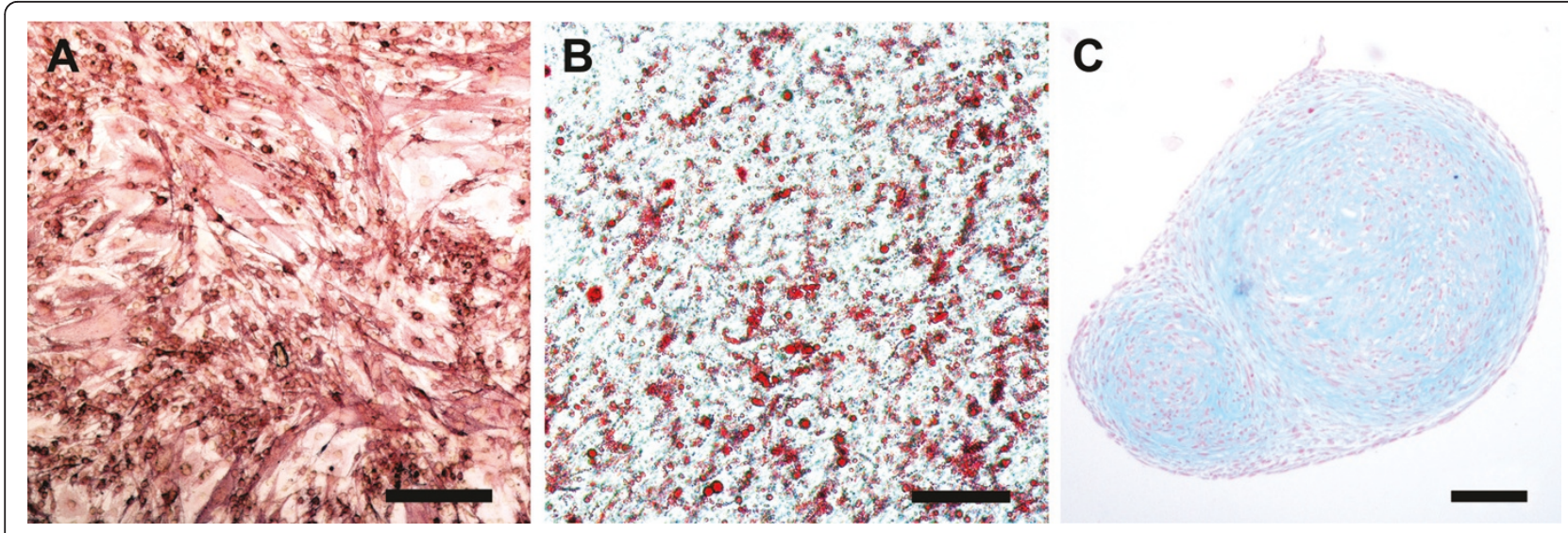

Figure 1 Representative images showing the multilineage differentiation potential of hASCs cultured in RegES medium. ALP staining for the osteogenic differentiation (A), Oil Red $O$ staining for the adipogenic differentiation (B), and Alcian Blue staining for the chondrogenic differentiation (C) of hASCs. Scale bar $500 \mu \mathrm{m}$ in (A), and $100 \mu \mathrm{m}$ in (B) and (C). ALP, alkaline phosphatase; hASCs, human adipose stem cells.

indicating the presence of sulphated GAGs typical to cartilage.

\section{Preliminary screening of the osteogenic medium compositions}

Preliminary screening was conducted to test a hypothesis if OM with low Dex and high AsA2-P concentrations could be efficient osteo-inducer of hASCs in HSbased medium (Additional file 2). According to the preliminary screening, it was concluded that AsA2-P is needed for the effective osteogenic differentiation of hASCs, and higher concentrations of AsA2-P resulted in increased runx2 expression and ALP activity (data not shown). Dex was found to suppress proliferation and collagen type I expression at high concentration (100 $\mathrm{nM})$, whereas low Dex concentration $(5 \mathrm{nM})$ was beneficial for the osteogenic differentiation when combined with high AsA2-P concentration $(250 \mu \mathrm{M})$. Consequently, the highest proliferation, ALP activity and runx2 expression were achieved with $150 \mu \mathrm{M}$ AsA2-P and $10 \mathrm{nM}$ Dex (OM2), and $250 \mu \mathrm{M}$ AsA2-P and $5 \mathrm{nM}$ Dex (OM3), compositions that were selected for further experiments to be compared with the traditionally used composition (OM1), containing $50 \mu \mathrm{M}$ AsA2-P and 100 nM Dex.

\section{Morphology and cell number}

Cell number was analyzed quantitatively at 7- and 14day time points (Figure 2) along with observation of morphology (Figure 3 and Additional file 3). Cell number was also analyzed at day 1 to test for equal plating efficiency; this was confirmed by the similar cell numbers between FBS, HS and RegES conditions at day 1 (data not shown). All different maintenance media were able to support the proliferation of hASCs as the cell numbers increased significantly $(P<0.05)$ with time when comparing day 1 and 14 within each serum condition. However, FBS MM and HS MM exhibited notably lower cell numbers than RegES MM (Figure 2). The increased growth rate of hASCs cultured in RegES MM was evident already after 7 days of culture, as was shown by cell number (Figure 2) and morphology analysis (Additional file 3). The cell number of RegES maintenance culture was significantly higher at day 14 ( $P$ $<0.05)$ when compared to HS and FBS maintenance cultures.

All osteogenic media (OM1-3) increased cell number significantly when compared to the respective MM under HS and RegES conditions at day 14 (Figure 2). Although a similar trend could be detected in FBS medium at day 14 in Figure 2 and Figure 3, the difference between $\mathrm{MM}$ and $\mathrm{OM}$ compositions was not significant in the quantitative analysis. In contrast, the stimulating effect of OM in HS and RegES cultures was shown already at day 7; OM3 exhibited significantly higher cell number than OM1 and MM in both HS and RegES. At day 14, in HS cultures the cell number was significantly higher in OM2 and OM3 when compared to OM1 and MM. In RegES cultures all OM compositions resulted in higher cell number than MM at the 14-day time point, but there were no differences between OM1, OM2 or OM3. This may be due to the high growth rate of hASCs in RegES medium; the cells had already reached confluency by day 14 and grew in multiple layers (Figure 3).

\section{Alkaline phosphatase activity}

The ALP activity of each sample was normalized to the amount of DNA in the sample, and all the results were considered relative to FBS MM at the 7-day time point. Due to notable donor variation, ALP activity is presented as a scatter plot showing the response of 

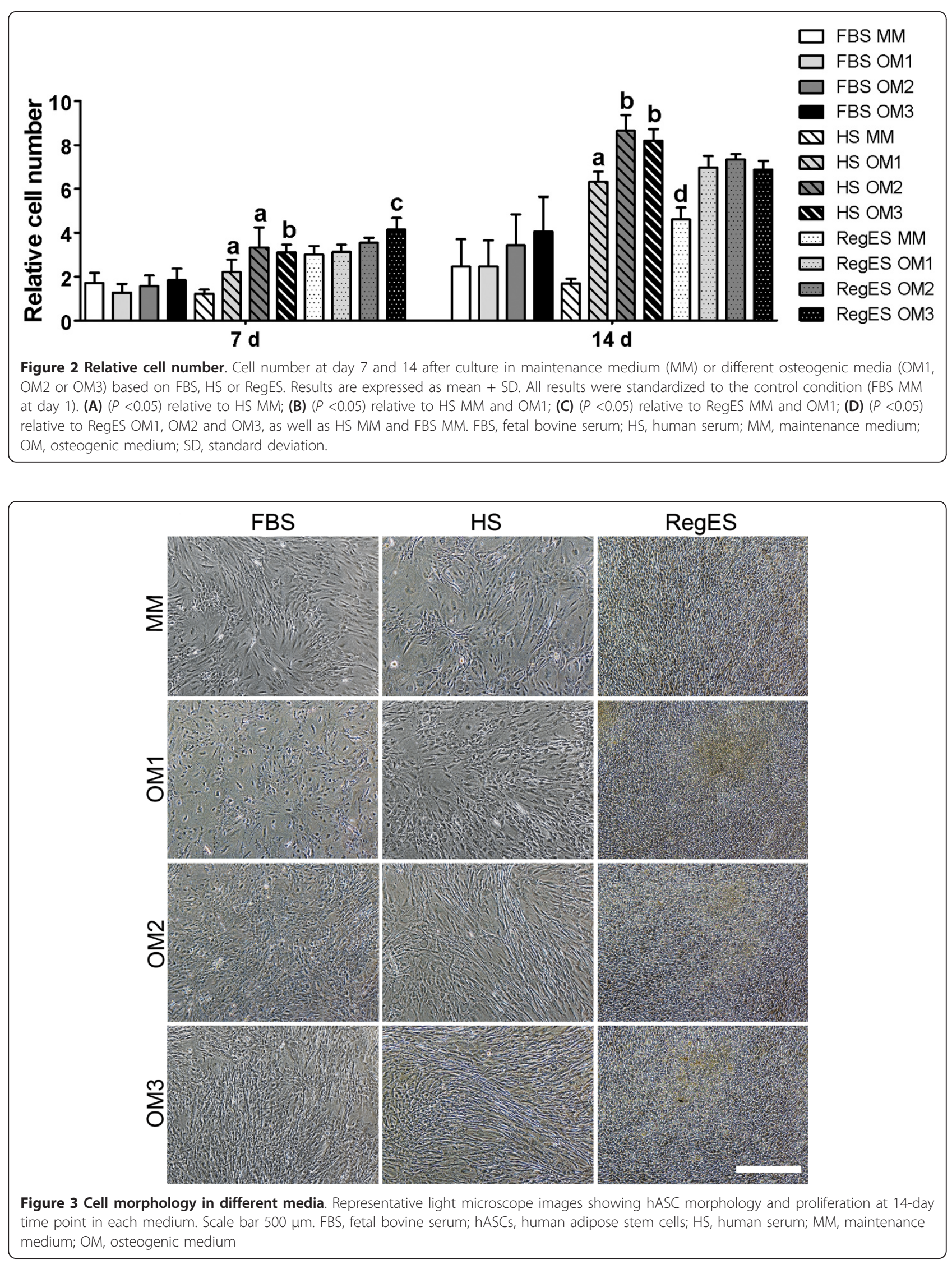


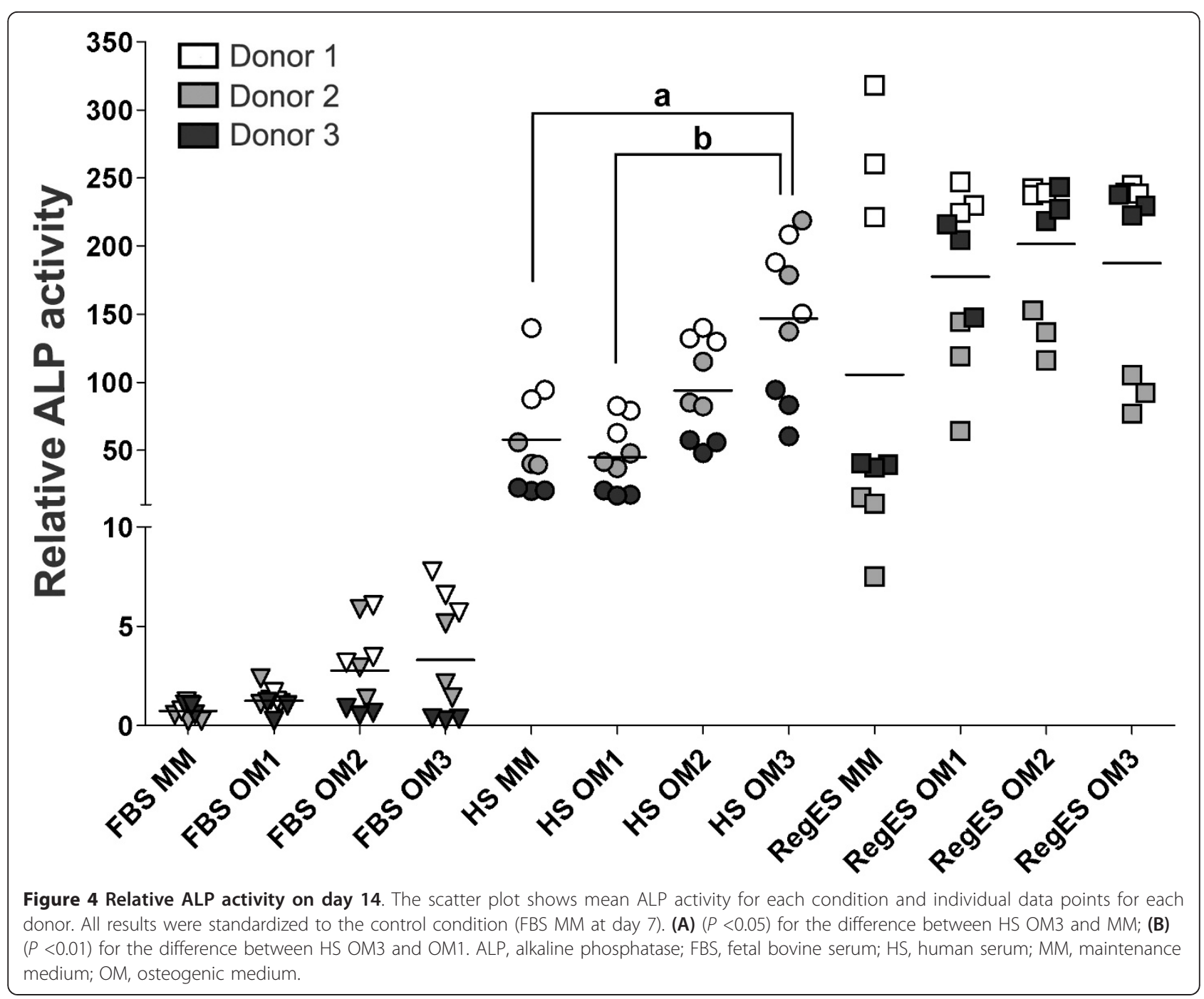

individual donors at day 14 (Figure 4). There were no statistically significant differences at day 7 (data not shown). At day 14, the ALP activity was notably low in all FBS cultures when compared to HS and RegES cultures. Two out of three donors exhibited a notable increase of ALP activity in response to OM2 and OM3 under FBS and HS conditions, whereas one donor did not seem to respond to any of the OM compositions. In RegES medium, the osteogenic supplements resulted in higher ALP activity with hASCs from two donors, whereas one donor line was induced by the RegES MM. Irrespective of the donor variation, the mean ALP activity of HS OM3 was significantly higher than with HS MM $(P=0.036)$ and OM1 $(P=0.006)$.

\section{Mineralization}

Similar to the ALP activity, OM3 increased mineralization the most in FBS cultures at day 14, but the difference to MM was not significant (Figure 5). Overall, mineralization was very weak under FBS conditions (Figures 5 and 6). In HS cultures, OM2 and OM3 resulted in a significantly higher mineralization than MM and OM1 at the 14-day time point. Although the difference between OM2 and OM3 in HS was not significant in the quantitative analysis of mineralization, the qualitative Alizarin Red staining showed more intense mineralization by OM3 than OM2 (Figure 6). In RegES cultures, all OM compositions induced significantly higher mineralization than RegES MM after 14 days of culture (Figures 5 and 6), but there were no significant differences between the different OM (Figure 5).

\section{Expression of osteogenic markers}

Quantitative RT-PCR was used to detect relative changes in the expression of osteogenic marker genes runx2A, DLX5, collagen type I, osteocalcin, and ALP (Figure 7). The relative expression of runx2A, one of the key osteogenic transcription factors, was significantly 

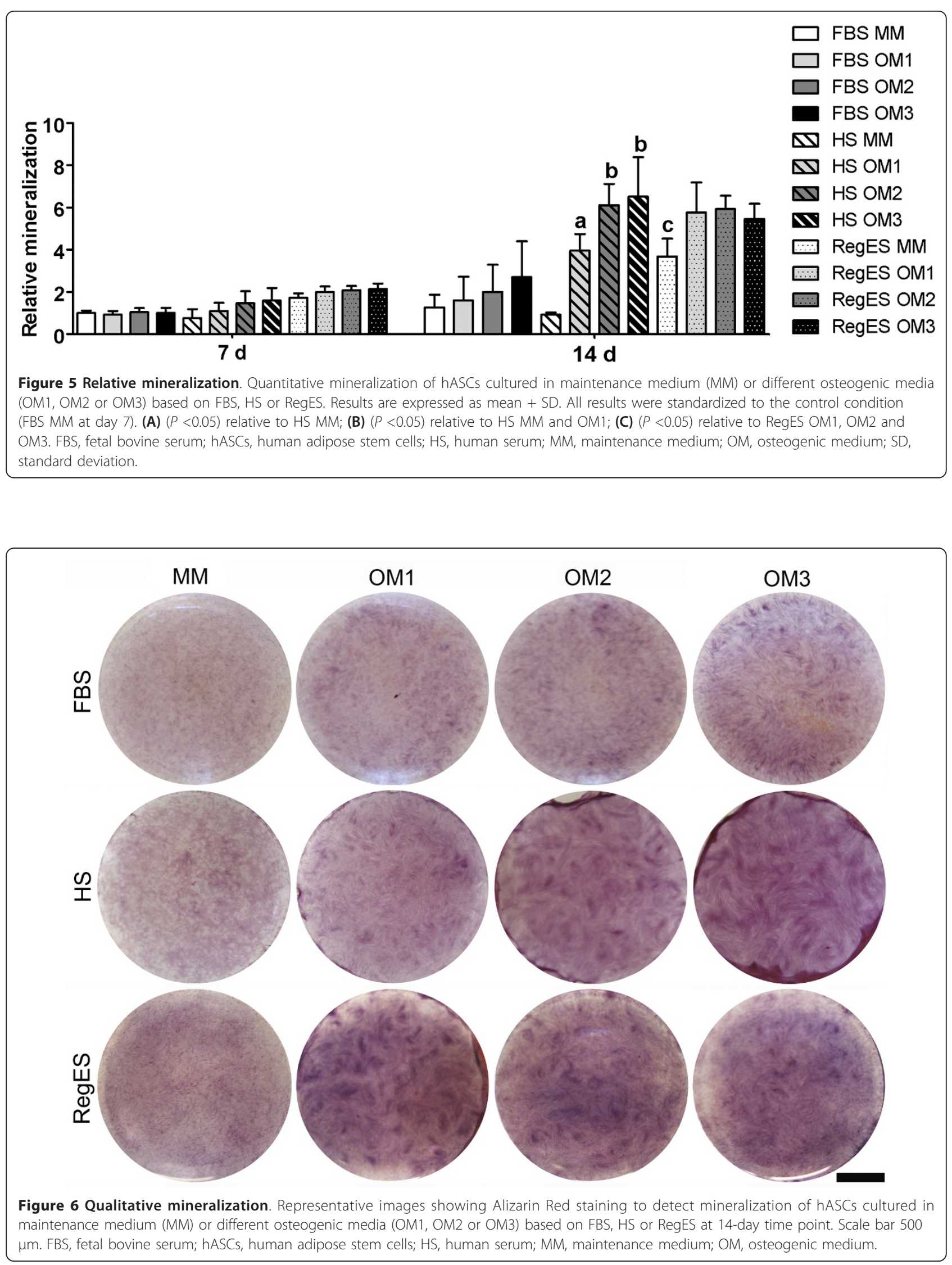

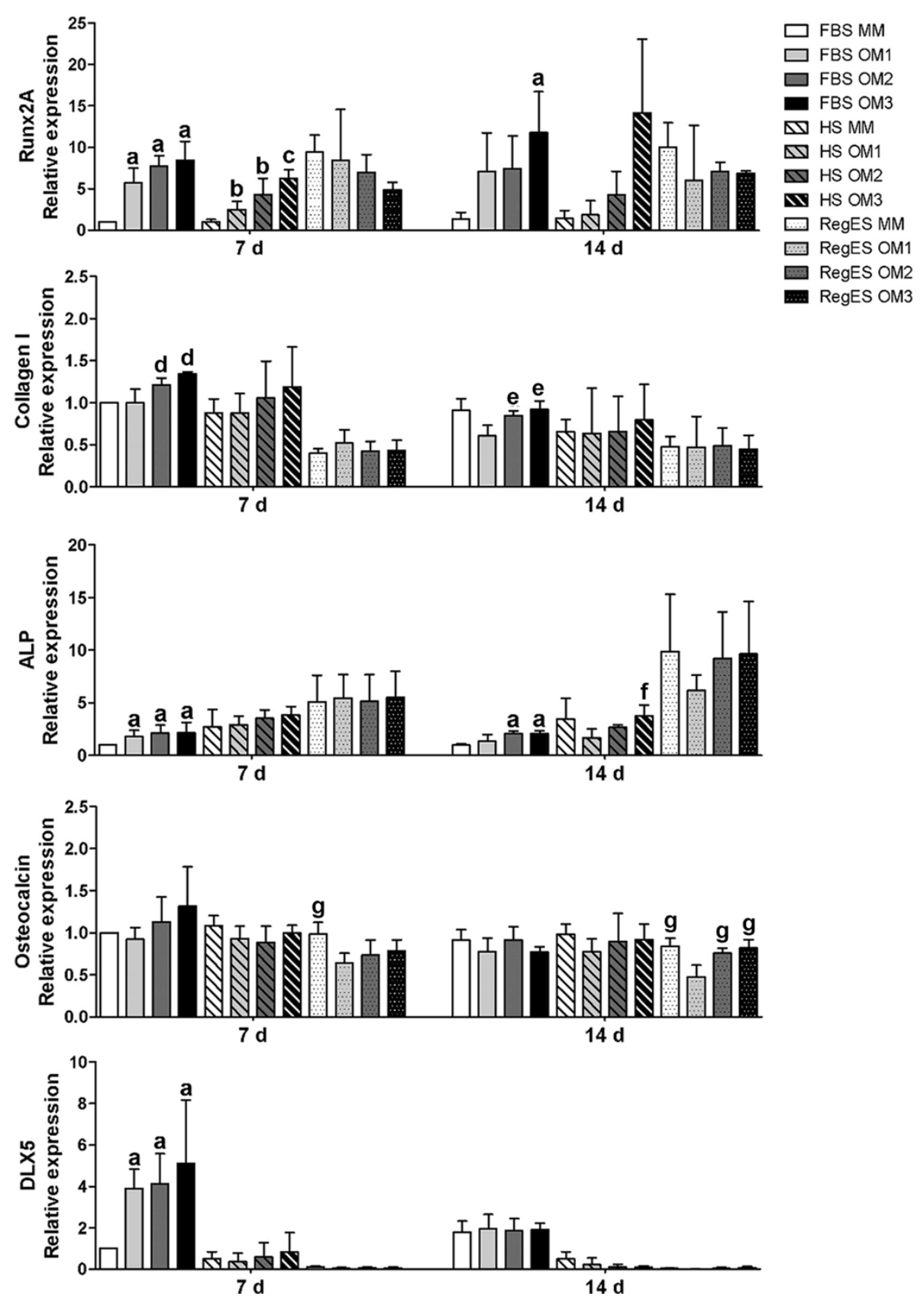

Figure 7 Quantitative real-time PCR results. The relative expression of osteogenic genes in hASCs cultured in maintenance medium (MM) or different osteogenic media (OM1, OM2 or OM3) based on FBS, HS or RegES at 7- and 14-day time points. Results are expressed as mean + SD. All results were standardized to the control condition (FBS MM at day 7). (A) $(P<0.05)$ relative to FBS $\mathrm{MM}$; $(\mathbf{B})(P<0.05)$ relative to HS MM; $(\mathbf{C})(P$ $<0.05)$ relative to HS MM and OM1; (D) $(P<0.05)$ relative to FBS MM and OM1; (E) $(P<0.05)$ relative to FBS OM1; $(\mathbf{F})(P<0.05)$ relative to HS OM1 and OM2; (G) $(P<0.05)$ relative to RegES OM1. FBS, fetal bovine serum; hASCs, human adipose stem cells; HS, human serum; MM, maintenance medium; OM, osteogenic medium; SD, standard deviation.

induced by the osteogenic components in FBS and HS conditions. In FBS cultures, all OM compositions induced significantly higher expression of runx2A than $\mathrm{MM}$ at the 7-day time point. At day 14, FBS OM3 ( $P=$ 0.048 ) showed significantly higher expression of runx2A
mRNA when compared to FBS MM. In HS cultures, all OM compositions exhibited significantly higher runx2A expression than HS MM $(P=0.024)$ at day 7 , and OM3 upregulated the runx2A expression significantly when compared to OM1. At day 14, a high expression of 
runx2A was achieved by HS OM3, but due to donor variation there was no significant difference to HS MM. In RegES cultures, there were no statistically significant differences between the OM compositions and MM for the expression of runx2A, likely due to the high basal level of runx2 expression in RegES MM. The expression of DLX5, activator of runx2, was significantly upregulated by all OM compositions on day 7 under FBS conditions, when compared to FBS MM $(P=0.024)$. However, there were no significant differences on day 14. The level of DLX5 expression was low under HS and RegES conditions.

The expression of collagen type I, a major component of organic bone matrix, was significantly increased by OM2 and OM3 under FBS conditions, specifically at day 7 when compared to both MM and OM1. At day 14, the expression of collagen type I level was slightly lower, but yet the expression was significantly higher with OM2 and OM3 than OM1 $(P=0.024)$. There were no significant differences between the groups under HS and RegES conditions. The level of collagen type I mRNA was generally lower in RegES than in FBS or HS conditions.

In contrast to collagen, the expression of ALP was notably upregulated in RegES MM, OM2 and OM3 cultures when compared to FBS and HS cultures at the 14day time point. Overall, the level of ALP expression was low in FBS medium. Nevertheless, significantly higher expression of ALP mRNA was detected in all FBS OM compositions at day 7 , and by OM2 and OM3 at day 14 , when compared to FBS MM $(P=0.024)$. In HS medium, OM3 showed significantly higher level of ALP expression at day 14 than OM1 $(P=0.024)$ and OM2 $(P=0.048)$, but not when compared to HS MM. Again there were no significant differences between RegES groups.

The expression of the late osteogenic marker osteocalcin was moderate in all of the cell cultures. Mild upregulation of osteocalcin expression by OM3 could be seen in FBS cultures at day 7, but the difference was not statistically significant. The elevated expression of osteocalcin in FBS OM3 was decreased to basal level by day 14. In RegES medium, the osteocalcin mRNA was downregulated by OM1, especially at the 14-day time point, where the level of osteocalcin in OM1 was significantly lower than in all the other RegES groups.

\section{Discussion}

While the effects of the OM supplements have been largely studied with BMSCs [15,19-21], there are only limited studies with ASCs $[16,22,39]$. One of the major shortcomings in all of these in vitro studies has been the lack of comparison between different serum conditions. Due to quality and safety issues, the clinical hASC-based applications need to move from the animal-derived products to human-derived or more preferably to defined and xeno-free conditions [24]. However, most of the in vitro studies are still conducted using FBS. Given that the serum conditions can significantly affect the cell response, it is crucial to obtain research data with more clinical relevance $[26,31]$.

Accordingly, we aimed to optimize the osteogenic culturing conditions for the in vitro induction of hASCs by testing different concentrations of Dex and AsA2-P in HS-based medium as well as in a defined, xeno-free medium RegES, with FBS medium functioning as a control. As hypothesized, the differential effect of FBS, HS or RegES media was evident in hASC proliferation and osteogenic differentiation. Comparison of the cell growth in FBS, HS and RegES maintenance media revealed a slightly higher growth rate in hASCs cultured in FBS MM than in HS MM, whereas the highest growth rate was achieved in RegES MM. When comparing $\mathrm{MM}$ and $\mathrm{OM}$ conditions in general, significantly higher cell number was achieved in OM than MM particularly in HS and RegES cultures. The effect of OM in cell number was also dependent of serum conditions; under FBS conditions OM3 with the highest AsA2-P concentration promoted cell growth most, but under HS conditions both OM2 and OM3 resulted in equally elevated cell numbers. High AsA2-P concentration (250 $\mu \mathrm{M})$ has been reported to stimulate proliferation of BMSCs and osteoblast-like cells [21,40], whereas high Dex concentration (100 nM) may inhibit proliferation [22]. More importantly, high AsA2-P concentration may stimulate proliferation without a reciprocal loss of differentiation potency [21,41].

The osteogenic induction capacity of OM1, OM2 and OM3 was compared by analyzing the ALP activity, mineralization and relative expression of several osteogenic markers. Contrary to expectations, the level of osteogenic differentiation was the lowest in FBS-cultured hASCs as measured by ALP activity and mineralization. Another significant finding was that the RegES $\mathrm{MM}$ alone was found to induce the early osteogenic differentiation as shown by the elevated ALP activity, although supplementation with Dex, AsA2-P and $\beta$-GP was required to achieve mineralization. On the whole, the inductive effect of high AsA2-P and low Dex concentration, as in OM3, was most evident in HS cultures, resulting in high ALP activity and mineralization. Prior studies have noted the importance of AsA2-P in the osteogenic differentiation of BMSCs and osteoblastic cells [21,41-43]. In contrast, Dex in high concentrations has been shown to inhibit osteogenic differentiation $[44,45]$, although it seems to be necessary for the efficient osteo-induction of MSCs in low concentrations $[15,19,20,44]$. 
In the present study, variation between donors could be detected particularly in ALP activity. Under HS and FBS conditions, two out of three donors exhibited a notable increase in ALP activity in response to OM2 and OM3, whereas one donor did not seem to respond to the OM supplements. Donor sample variability affects the interpretation of the results regardless of analyzing method and diminishes the statistical significance by increasing standard deviation. Others have established this problem previously with ASCs $[11,23,46]$ and BMSCs $[15,47,48]$. With BMSCs, Jaiswal and co-workers showed that the basal level of ALP activity as well as the timing of the peak ALP activity varied greatly between the different donor samples irrespective of donor age [15]. The relative fold induction in ALP activity varied 1.5- to 6.4-fold depending on donor [15]. Similar kind of variation has been detected with ASCs as well $[11,23,46]$.

The osteogenic effect of OM1, OM2 and OM3 under different serum conditions was further studied by analyzing the relative expression of osteogenic markers, runx2A, DLX5, collagen type I, osteocalcin and ALP. Overall, the qRT-PCR data demonstrated significant upregulation of runx2A mRNA under osteogenic differentiation in FBS and HS medium, and early stimulation of DLX5 under FBS conditions. The regulation of other markers, collagen type I, osteocalcin and ALP, was modest. When comparing the different OM compositions, $\mathrm{OM} 2$ and OM3 resulted in significantly higher expression of runx $2 \mathrm{~A}$, collagen type I, and ALP, than corresponding MM under FBS or HS conditions. In some cases, OM2 and OM3 also resulted in higher expression of runx2A, collagen type I, and ALP than OM1. Under FBS conditions, OM3 induced significantly higher expression of runx2A when compared to FBS MM at day 14 . In addition, collagen type I expression was significantly upregulated by FBS OM2 and OM3 at day 7 in comparison to both MM and OM1, and versus OM1 on day 14 . Under HS conditions, OM3 resulted in significantly higher runx2A expression when compared to HS MM and OM1 at day 7. Moreover, HS OM3 resulted in higher level of ALP expression than OM1 and OM2 on day 14. Hence, greater expression of osteogenic markers can be achieved by OM with increased AsA2-P and lowered Dex, that is OM2 or OM3 composition.

In RegES medium, significant differences in the runx2A, collagen type I or ALP expression were not detected, likely due to the high basal level of expression in RegES MM. However, the level of osteocalcin mRNA was significantly lower in RegES OM1 than in MM, OM2 or OM3 at day 14. The expression of DLX5 appeared to peak already on day 7 under osteogenic induction under FBS conditions, whereas no regulation of DLX5 was detected under HS and RegES conditions.

There were also notable differences in the collagen type I and ALP mRNA expression between the serum conditions in general. The expression of collagen type I was notably lower in all RegES groups when compared to the respective FBS and HS groups. The expression of ALP, on the contrary, was higher in the RegES groups than in FBS and HS groups. This result correlates with the high ALP protein activity in RegES cultures, but in turn, not with the high ALP activity found in HS cultures. Unexpectedly, the expression of runx2, collagen type I, ALP or osteocalcin was not upregulated by time (day 7 versus 14) in any of the OM groups. Although we did not see significant upregulation by time, the culture period of 14 days has been shown to be sufficient for the detection of osteogenic gene expression [49-51]. Furthermore, considering clinical applications, the in vitro culture period should be minimized, as prolonged culture may increase the risk of contamination or genetic abnormalities. Therefore, the inductive effects in vitro should also appear within as short a time period as possible.

In contrast to ALP activity and mineralization, relatively small changes were detected in mRNA expression levels between MM and OM groups under all serum conditions. Although challenging the common conception, the lack of upregulation or even downregulation of certain osteogenic markers in ASCs upon osteogenic differentiation has been reported previously [12,18]. For OM-induced BMSCs, similar differences have been reported between the data obtained on protein level and real-time PCR for collagen type I expression [52]. The flow cytometric analysis showed significantly upregulated expression of collagen type I in the ECM of differentiated BMSCs, but there was no notable increase in collagen type I mRNA even after osteogenic differentiation according to real-time PCR [52]. While this phenomenon is still poorly understood, it is evident that the route of mRNA to protein is a highly regulated and complex pathway, where even small changes at transcriptional or posttranscriptional level can have a major phenotypic effect $[12,18,53]$.

One of the main scopes of the present study was to investigate whether xeno-free RegES medium could be utilized for the efficient osteogenic differentiation of hASCs. Taken together, hASCs cultured in RegES showed increased osteogenic capacity, an effect likely explained by the composition of the RegES medium (Additional file 1). Apart from the several growth factors and cytokines, the high concentration of AsA2-P in RegES MM $(50 \mu \mathrm{g} / \mathrm{ml}$ or $170 \mu \mathrm{M})$ may account for the high basal level of cell growth and osteogenic differentiation of hASCs in the 
plain RegES medium. Taking into account the additional AsA2-P in the osteogenic media, the concentrations in RegES OM1, OM2 and OM3, raised to 220, 320, and 420 $\mu \mathrm{M}$, respectively. The maximal advantage in growth rate and differentiation in RegES-based osteogenic media was reached by AsA2-P concentrations varying from 220 to $320 \mu \mathrm{M}$, correlating to the results obtained with OM3 under FBS and HS conditions. However, as already mentioned, supplementation with Dex and $\beta$-GP in addition to AsA2-P was required for efficient osteo-induction and maturation of hASCs cultured in RegES.

\section{Conclusions}

In summary, our results show that the serum conditions have a significant effect on the hASC behavior, such as proliferation and osteogenic differentiation capacity. Osteogenic differentiation of hASCs was the weakest in FBS-based medium. The plain RegES medium was able to induce early osteogenic differentiation of hASCs, although supplementation with Dex, AsA2-P and $\beta$-GP was needed to achieve mineralization. One of the key findings was that the commonly used OM1 supports the in vitro osteo-induction of hASCs poorly in FBS and HS medium. Instead, OM with higher AsA2-P and lower Dex should be used for the osteogenic differentiation of hASCs under FBS and HS conditions.

\section{Additional material}

Additional file 1: RegES medium. Complete formulation for RegES medium.

Additional file 2: Preliminary screening. Osteogenic medium (OM) compositions of the preliminary screening (all in HS medium).

Additional file 3: Cell morphology at different time points. Light microscope images showing representative hASC morphology in each maintenance medium at 3-, 7- and 14-day time points. Scale bar $500 \mu \mathrm{m}$.

\begin{abstract}
Abbreviations
ALP: alkaline phosphatase; AsA2-P: L-ascorbic acid 2-phosphate; $\beta$-GP: betaglycerophosphate; BMP: bone morphogenetic protein; BMSC: bone marrowderived mesenchymal stem cell; Dex: dexamethasone; FBS: fetal bovine serum; GAGs: glycosaminoglycans; hASC: human adipose stem cell; HS: human serum; MM: maintenance medium; MSC: mesenchymal stem cell; OM: osteogenic medium; PFA: paraformaldehyde; qRT-PCR: quantitative realtime reverse transcription polymerase chain reaction.
\end{abstract}

\section{Authors' contributions}

LK carried out most of the cell culture studies and data analysis, drafted the manuscript, and participated in manuscript editing. SH designed and coordinated the study, had a major contribution in interpretation of data, and participated in writing and editing of the manuscript. BM carried out the flow cytometric analysis, participated in the data analysis and manuscript editing. $\mathrm{HH}$ carried out the statistical analysis and contributed to the interpretation of data. KR and HS conceived the RegES medium, and helped to draft and edit the manuscript. GS participated in the study design, coordination, and manuscript editing. SM participated in the study design and coordination, contributed to the interpretation of data, and participated in manuscript editing. All authors read and approved the final manuscript.

\section{Competing interests}

The authors KR and HS declare competing financial interests defined as patent application relating to the xeno-free culture medium RegES.

\section{Acknowledgements}

The authors thank Dr. Hannu Kuokkanen for the delivery of fat samples for stem cell isolation, and Anna-Maija Honkala, Miia Juntunen, Sari Kalliokoski, and Minna Salomäki for technical assistance with hASCs. We thank Miia Juntunen for the preliminary experiments. We also thank Outi Melin and Elina Konsén for preparing the RegES medium. Special thanks to Vitrolife AB for valuable comments. This study has been financially supported by the Competitive Research Funding of Tampere University Hospital (grants 9L057, 9K117, 9L100, 9M058 and 9J014), the Finnish Funding Agency for Technology and Innovation (TEKES), the Academy of Finland, and the Science Centre of Tampere City.

\section{Author details}

${ }^{1}$ Adult Stem Cells Group, Institute of Biomedical Technology, University of Tampere, Biokatu 8, Tampere Fl-33014, Finland. 'BioMediTech, Biokatu 10, Tampere FI-33520, Finland. ${ }^{3}$ Science Center, Tampere University Hospital, Biokatu 10, Tampere Fl-33521, Finland. ${ }^{4}$ Department of Biomaterials Science and Technology, University of Twente, De Horst 2, Enchede 7522 LW, The Netherlands. ${ }^{5}$ Tampere School of Public Health, University of Tampere, Medisiinarinkatu 3, Tampere FI-33014, Finland. ${ }^{6}$ Cardiology Group, Institute of Biomedical Technology, University of Tampere, Biokatu 8, Tampere Fl-33014, Finland. ${ }^{7}$ Ophthalmology Group, Institute of Biomedical Technology, University of Tampere, Biokatu 8, Tampere Fl-33014, Finland. ${ }^{8}$ Department of Oral and Maxillofacial Surgery, Institute of Dentistry, University of Oulu, Aapistie 3, Oulu FI-90014, Finland.

Received: 29 September 2012 Revised: 3 January 2013

Accepted: 4 February 2013 Published: 15 February 2013

\section{References}

1. Mesimaki K, Lindroos B, Tornwall J, Mauno J, Lindqvist C, Kontio R, Miettinen $S$, Suuronen R: Novel maxillary reconstruction with ectopic bone formation by GMP adipose stem cells. Int J Oral Maxillofac Surg 2009, 38:201-209.

2. Thesleff T, Lehtimaki K, Niskakangas T, Mannerstrom B, Miettinen S, Suuronen R, Ohman J: Cranioplasty with adipose-derived stem cells and biomaterial. A novel method for cranial reconstruction. Neurosurgery 2011, 68:1535-1540.

3. Zuk PA, Zhu M, Mizuno H, Huang J, Futrell JW, Katz AJ, Benhaim P, Lorenz HP, Hedrick MH: Multilineage cells from human adipose tissue: implications for cell-based therapies. Tissue Eng 2001, 7:211-228.

4. Knippenberg M, Helder MN, Zandieh Doulabi B, Wuisman PI, Klein-Nulend J: Osteogenesis versus chondrogenesis by BMP-2 and BMP-7 in adipose stem cells. Biochem Biophys Res Commun 2006, 342:902-908.

5. Al-Salleeh F, Beatty MW, Reinhardt RA, Petro TM, Crouch L: Human osteogenic protein-1 induces osteogenic differentiation of adiposederived stem cells harvested from mice. Arch Oral Biol 2008, 53:928-936.

6. Song I, Kim BS, Kim CS, Im Gl: Effects of BMP-2 and vitamin D3 on the osteogenic differentiation of adipose stem cells. Biochem Biophys Res Commun 2011, 408:126-131.

7. Garrison KR, Donell S, Ryder J, Shemilt I, Mugford M, Harvey I, Song F: Clinical effectiveness and cost-effectiveness of bone morphogenetic proteins in the non-healing of fractures and spinal fusion: a systematic review. Health Technol Assess 2007, 11:1-150, iii-iv.

8. Wysocki RW, Cohen MS: Ectopic ossification of the triceps muscle after application of bone morphogenetic protein-7 to the distal humerus for recalcitrant nonunion: a case report. J Hand Surg Am 2007, 32:647-650.

9. Axelrad TW, Steen B, Lowenberg DW, Creevy WR, Einhorn TA: Heterotopic ossification after the use of commercially available recombinant human bone morphogenetic proteins in four patients. J Bone Joint Surg Br 2008, 90:1617-1622.

10. Alarmo EL, Parssinen J, Ketolainen JM, Savinainen K, Karhu R, Kallioniemi A: BMP7 influences proliferation, migration, and invasion of breast cancer cells. Cancer Lett 2009, 275:35-43.

11. Zuk P, Chou YF, Mussano F, Benhaim P, Wu BM: Adipose-derived stem cells and BMP2: part 2. BMP2 may not influence the osteogenic fate of human adipose-derived stem cells. Connect Tissue Res 2011, 52:119-132. 
12. Egusa H, lida K, Kobayashi M, Lin TY, Zhu M, Zuk PA, Wang CJ, Thakor DK, Hedrick MH, Nishimura I: Downregulation of extracellular matrix-related gene clusters during osteogenic differentiation of human bone marrowand adipose tissue-derived stromal cells. Tissue Eng 2007, 13:2589-2600.

13. Giusta MS, Andrade H, Santos AV, Castanheira P, Lamana L, Pimenta AM, Goes AM: Proteomic analysis of human mesenchymal stromal cells derived from adipose tissue undergoing osteoblast differentiation. Cytotherapy 2010, 12:478-490

14. De Ugarte DA, Morizono K, Elbarbary A, Alfonso Z, Zuk PA, Zhu M, Dragoo JL, Ashjian P, Thomas B, Benhaim P, Chen I, Fraser J, Hedrick MH: Comparison of multi-lineage cells from human adipose tissue and bone marrow. Cells Tissues Organs 2003, 174:101-109.

15. Jaiswal N, Haynesworth SE, Caplan Al, Bruder SP: Osteogenic differentiation of purified, culture-expanded human mesenchymal stem cells in vitro. J Cell Biochem 1997, 64:295-312.

16. de Girolamo L, Sartori MF, Albisetti W, Brini AT: Osteogenic differentiation of human adipose-derived stem cells: comparison of two different inductive media. J Tissue Eng Regen Med 2007, 1:154-157.

17. Hennig T, Lorenz H, Thiel A, Goetzke K, Dickhut A, Geiger F, Richter W: Reduced chondrogenic potential of adipose tissue derived stromal cells correlates with an altered TGFbeta receptor and BMP profile and is overcome by BMP-6. J Cell Physiol 2007, 211:682-691.

18. Shafiee A, Seyedjafari E, Soleimani M, Ahmadbeigi N, Dinarvand P, Ghaemi N: A comparison between osteogenic differentiation of human unrestricted somatic stem cells and mesenchymal stem cells from bone marrow and adipose tissue. Biotechnol Lett 2011, 33:1257-1264.

19. Coelho MJ, Fernandes MH: Human bone cell cultures in biocompatibility testing. Part II: effect of ascorbic acid, beta-glycerophosphate and dexamethasone on osteoblastic differentiation. Biomaterials 2000, 21:1095-1102

20. Atmani H, Audrain C, Mercier L, Chappard D, Basle MF: Phenotypic effects of continuous or discontinuous treatment with dexamethasone and/or calcitriol on osteoblasts differentiated from rat bone marrow stromal cells. J Cell Biochem 2002, 85:640-650.

21. Choi KM, Seo YK, Yoon HH, Song KY, Kwon SY, Lee HS, Park JK: Effect of ascorbic acid on bone marrow-derived mesenchymal stem cell proliferation and differentiation. J Biosci Bioeng 2008, 105:586-594.

22. Malladi $P, X u Y$, Yang GP, Longaker MT: Functions of vitamin $D$, retinoic acid, and dexamethasone in mouse adipose-derived mesenchymal cells. Tissue Eng 2006, 12:2031-2040.

23. Bieback K, Hecker A, Schlechter T, Hofmann I, Brousos N, Redmer T, Besser D, Kluter H, Muller AM, Becker M: Replicative aging and differentiation potential of human adipose tissue-derived mesenchymal stromal cells expanded in pooled human or fetal bovine serum. Cytotherapy 2012, 14:570-583.

24. Gstraunthaler $\mathrm{G}$ : Alternatives to the use of fetal bovine serum: serum-free cell culture. Altex 2003, 20:275-281.

25. Le Blanc K, Samuelsson H, Lonnies L, Sundin M, Ringden O: Generation of immunosuppressive mesenchymal stem cells in allogeneic human serum. Transplantation 2007, 84:1055-1059.

26. Lindroos B, Boucher $S$, Chase L, Kuokkanen $H$, Huhtala H, Haataja R, Vemuri M, Suuronen $\mathrm{R}$, Miettinen S: Serum-free, xeno-free culture media maintain the proliferation rate and multipotentiality of adipose stem cells in vitro. Cytotherapy 2009, 11:958-972.

27. Lund P, Pilgaard L, Duroux M, Fink T, Zachar V: Effect of growth media and serum replacements on the proliferation and differentiation of adipose-derived stem cells. Cytotherapy 2009, 11:189-197.

28. Rajala K, Lindroos B, Hussein SM, Lappalainen RS, Pekkanen-Mattila M Inzunza J, Rozell B, Miettinen S, Narkilahti S, Kerkela E, Aalto-Setala K, Otonkoski T, Suuronen R, Hovatta O, Skottman H: A defined and xeno-free culture method enabling the establishment of clinical-grade human embryonic, induced pluripotent and adipose stem cells. PLOS One 2010, 5:e10246.

29. Gimble J, Guilak F: Adipose-derived adult stem cells: isolation, characterization, and differentiation potential. Cytotherapy 2003, 5:362-369.

30. Dominici M, Le Blanc K, Mueller I, Slaper-Cortenbach I, Marini F, Krause D, Deans R, Keating A, Prockop D, Horwitz E: Minimal criteria for defining multipotent mesenchymal stromal cells. The International Society for Cellular Therapy position statement. Cytotherapy 2006, 8:315-317.

31. Lindroos B, Aho KL, Kuokkanen H, Raty S, Huhtala H, Lemponen R, YliHarja O, Suuronen R, Miettinen S: Differential gene expression in adipose stem cells cultured in allogeneic human serum versus fetal bovine serum. Tissue Eng Part A 2010, 16:2281-2294

32. Tirkkonen $L$, Halonen $H$, Hyttinen J, Kuokkanen $H$, Sievanen $H$, Koivisto AM, Mannerstrom B, Sandor GK, Suuronen R, Miettinen S, Haimi S: The effects of vibration loading on adipose stem cell number, viability and differentiation towards bone-forming cells. J R Soc Interface 2011, 8:1736-1747

33. Komori T: Regulation of bone development and extracellular matrix protein genes by RUNX2. Cell Tissue Res 2010, 339:189-195.

34. Banerjee C, Javed A, Choi JY, Green J, Rosen V, van Wijnen AJ, Stein JL, Lian JB, Stein GS: Differential regulation of the two principal Runx2/Cbfa1 $\mathrm{n}$-terminal isoforms in response to bone morphogenetic protein-2 during development of the osteoblast phenotype. Endocrinology 2001, 142:4026-4039.

35. Gabrielsson BG, Olofsson LE, Sjogren A, Jernas M, Elander A, Lonn M, Rudemo M, Carlsson LM: Evaluation of reference genes for studies of gene expression in human adipose tissue. Obes Res 2005, 13:649-652.

36. Fink $T$, Lund $P$, Pilgaard $L$, Rasmussen JG, Duroux $M$, Zachar V: Instability of standard PCR reference genes in adipose-derived stem cells during propagation, differentiation and hypoxic exposure. BMC Mol Biol 2008 9:98.

37. Lind M: Growth factors: possible new clinical tools. A review. Acta Orthop Scand 1996, 67:407-417.

38. McIntosh K, Zvonic S, Garrett S, Mitchell JB, Floyd ZE, Hammill L, Kloster A, Di Halvorsen Y, Ting JP, Storms RW, Goh B, Kilroy G, Wu X, Gimble JM: The immunogenicity of human adipose-derived cells: temporal changes in vitro. Stem Cells 2006, 24:1246-1253.

39. Zhou YS, Liu YS, Tan JG: Is 1, 25-dihydroxyvitamin D3 an ideal substitute for dexamethasone for inducing osteogenic differentiation of human adipose tissue-derived stromal cells in vitro? Chin Med J (Engl) 2006, 119:1278-1286.

40. Maehata Y, Takamizawa S, Ozawa S, Izukuri K, Kato Y, Sato S, Lee MC, Kimura A, Hata R: Type III collagen is essential for growth acceleration of human osteoblastic cells by ascorbic acid 2-phosphate, a long-acting vitamin C derivative. Matrix Biol 2007, 26:371-381.

41. Takamizawa S, Maehata Y, Imai K, Senoo H, Sato S, Hata R: Effects of ascorbic acid and ascorbic acid 2-phosphate, a long-acting vitamin C derivative, on the proliferation and differentiation of human osteoblastlike cells. Cell Biol Int 2004, 28:255-265.

42. Torii Y, Hitomi K, Tsukagoshi N: L-ascorbic acid 2-phosphate promotes osteoblastic differentiation of MC3T3-E1 mediated by accumulation of type I collagen. J Nutr Sci Vitaminol (Tokyo) 1994, 40:229-238.

43. Hitomi $\mathrm{K}$, Torii $\mathrm{Y}$, Tsukagoshi $\mathrm{N}$ : Increase in the activity of alkaline phosphatase by L-ascorbic acid 2-phosphate in a human osteoblast cell line, HuO-3N1. J Nutr Sci Vitaminol (Tokyo) 1992, 38:535-544.

44. Wang H, Pang B, Li Y, Zhu D, Pang T, Liu Y: Dexamethasone has variable effects on mesenchymal stromal cells. Cytotherapy 2012, 14:423-430.

45. Malladi $P, X u Y$, Chiou M, Giaccia AJ, Longaker MT: Effect of reduced oxygen tension on chondrogenesis and osteogenesis in adipose-derived mesenchymal cells. Am J Physiol Cell Physiol 2006, 290:C1139-1146.

46. Guilak F, Lott KE, Awad HA, Cao Q, Hicok KC, Fermor B, Gimble JM: Clonal analysis of the differentiation potential of human adipose-derived adult stem cells. J Cell Physiol 2006, 206:229-237.

47. Phinney DG, Kopen G, Righter W, Webster S, Tremain N, Prockop DJ: Donor variation in the growth properties and osteogenic potential of human marrow stromal cells. J Cell Biochem 1999, 75:424-436.

48. Siddappa R, Licht $R$, van Blitterswijk C, de Boer J: Donor variation and loss of multipotency during in vitro expansion of human mesenchymal stem cells for bone tissue engineering. J Orthop Res 2007, 25:1029-1041.

49. Rada T, Santos TC, Marques AP, Correlo VM, Frias AM, Castro AG, Neves NM, Gomes ME, Reis RL: Osteogenic differentiation of two distinct subpopulations of human adipose-derived stem cells: an in vitro and in vivo study. J Tissue Eng Regen Med 2012, 6:1-11.

50. Vishnubalaji R, Al-Nbaheen M, Kadalmani B, Aldahmash A, Ramesh T: Comparative investigation of the differentiation capability of bonemarrow- and adipose-derived mesenchymal stem cells by qualitative and quantitative analysis. Cell Tissue Res 2012, 347:419-427.

51. Behr B, Tang C, Germann G, Longaker MT, Quarto N: Locally applied vascular endothelial growth factor a increases the osteogenic healing capacity of human adipose-derived stem cells by promoting osteogenic and endothelial differentiation. Stem Cells 2011, 29:286-296. 
52. Liu F, Akiyama Y, Tai S, Maruyama K, Kawaguchi Y, Muramatsu K, Yamaguchi K: Changes in the expression of CD106, osteogenic genes, and transcription factors involved in the osteogenic differentiation of human bone marrow mesenchymal stem cells. J Bone Miner Metab 2008, 26:312-320.

53. Zhang ZJ, Zhang H, Kang Y, Sheng PY, Ma YC, Yang ZB, Zhang ZQ, Fu M, He AS, Liao WM: miRNA expression profile during osteogenic differentiation of human adipose-derived stem cells. J Cell Biochem 2012, 113:888-898.

doi:10.1186/scrt165

Cite this article as: Kyllönen et al.: Effects of different serum conditions on osteogenic differentiation of human adipose stem cells in vitro. Stem Cell Research \& Therapy 2013 4:17.

\section{Submit your next manuscript to BioMed Central} and take full advantage of:

- Convenient online submission

- Thorough peer review

- No space constraints or color figure charges

- Immediate publication on acceptance

- Inclusion in PubMed, CAS, Scopus and Google Scholar

- Research which is freely available for redistribution

Submit your manuscript at www.biomedcentral.com/submit 